Article

\title{
Very Strange Sit-Coms: J. G. Ballard, Psychopathology, and Online Participatory Media
}

\author{
Martin Gleghorn
}

English Studies, Durham University, 77 Hallgarth House, Durham DH1 3AY, UK; j.m.gleghorn@durham.ac.uk

Received: 22 January 2019; Accepted: 4 March 2019; Published: 7 March 2019

\begin{abstract}
We're all going to be starring in our own sit-coms, and they'll be very strange sit-coms too, like the inside of our heads."- J. G. Ballard, Extreme Metaphors. Ballard's prediction about the possibility of projecting the inside of our own heads is highly illuminating in light of contemporary discourses on participatory media culture and online video-sharing platforms. This is not least due to the documented instances of violence and sexual deviance surrounding prominent figures on YouTube that lend a considerable amount of credence to what Ballard described, in his 1977 short story 'The Intensive Care Unit', as a 'liberating affectlessness [that] allowed those who wished to explore the fullest range of sexual possibility and paved the way for the day when a truly guilt-free sexual perversity and, even, psychopathology might be enjoyed by all.' This article examines how Ballard's preoccupation with this 'liberating affectlessness' - or as he notably termed it in his introduction to Crash, 'the death of affect' - compares to the impetus that psychologists such as Jonathan Rottenberg and Sheri L. Johnson place upon an 'affective science' approach to exploring and treating psychopathology - an approach that they affirm has 'tremendous potential to facilitate scientific work on the role of emotions in psychopathology.' This active interplay between emotion and affect (or the calculated lack of) on one hand, and psychopathology on the other that Ballard and Rottenberg and Johnson investigate from different angles also feeds into discourses on online participatory media and the ways that users engage with online media. Specifically, this section of the article will draw upon the roles of private and public spaces, and the breakdown of traditional barriers between them, as well as the commercial factors that define and underpin this new media culture. 'The Intensive Care Unit' and later novels such as Cocaine Nights (1996) play upon these themes as a means of anticipating and demonstrating how, in Ballard's fiction as well as in real-life instances, psychopathology emerges in the breakdown of the barriers between lives lived excessively on screen and the external, sensory and emotional world.
\end{abstract}

Keywords: Ballard; online participatory media; social media; YouTube; psychopathology; affect; emotion

\section{Introduction}

In 2013, the prominent news and entertainment site Buzzfeed posted an article with the sensationalist claim that J. G. Ballard had 'predicted social media' (Lewis 2013) back in 1977, in an article for Vogue entitled 'The Future of the Future'. The section of Ballard's essay Buzzfeed excitedly ran with as central to their claim featured visions of 'a computer trained to pick out only our best profiles, our wittiest dialogue, our most affecting expressions filmed through the kindest filters, and then stitch these together into a heightened re-enactment of the day (Ballard 1997a, p. 226).' While Ballard's prescience in this instance is both amusing and impressive, there is far at more at play in his thinking than an insightful prediction of surface-level, front-end mechanisms of social and online media such as the Facebook news feed, Instagram filters, YouTube vlogs and Snapchat stories. 
In his response to the Buzzfeed article, Simon Sellars riffs on the deliberate complexities of Ballard's theories through a close reading of the fictional counterpart to 'The Future of the Future': the 1977 short story, 'The Intensive Care Unit'. For Sellars, 'The Intensive Care Unit' encapsulates how:

'Ballard could expose the vacuity and never-attainable levels of satisfaction that power the logic of the consumerist engine, yet he was not above the thrill of it all, for he understood the liberating charge that comes from total abandonment. He saw the capturing, framing and enhancement of perversity by modern technology as beneficial, an unprecedented 'back door pass into the realm of psychopathology' (Sellars 2013).

Like Sellars, this article will offer a reading of 'The Intensive Care Unit'. However, it will do so through the lens of contemporary theories on affect and social media, as well as the role that what psychologists Jonathan Rottenberg and Sheri L. Johnson call 'affective science' plays in the understanding and treatment of various psychopathologies. In the case of the former, Tony D. Sampson, Darren Ellis and Stephen Maddison note that one of the fundamental challenges of examining affect and social media is that it 'is not as if the two can be easily separated-as if affect was something that circulates and penetrates social media, rather than constituting its very being (Sampson et al. 2018, p. 6)'.

By bringing in recent work in clinical psychology and the emerging area of affect and social media, this article seeks to offer new insights into Ballard's writing through the application of clinical work on psychopathology and emotion to it, an area that is perhaps not as extensive as one might expect. Exceptions to this, however, include Dan O'Hara's essay 'Reading Posture and Gesture in Ballard's Novels', and Andrzej Gasiorek's 2005 coda on Violence and Psychopathology. One of the most intriguing threads of Gasiorek's coda is how he captures Ballard's awareness of the gratuitous late twentieth-century violence that emerges when distinctions between literality and metaphor collapse-concluding that, what 'Ballard defends as the 'morally free psychopathology of metaphor [original emphasis], as an element in one's dreams', is, when carried over by those who literalise metaphor into the domain where it has no place, an id-driven psychopathology that lays waste to human life.' (Gasiorek 2005, p. 212). O'Hara, meanwhile, charts the interplay between psychopathology, technology and communication through the dual framework of Gregory Bateson's 1956 psychological concept of the double bind, and R. D. Laing's interpretation of that concept, namely its ubiquity in the everyday life of the 1960s. He concludes that Ballard's writing-especially his later fiction-reveals 'the metacommunicational contexts which we create, which conjoin inner space with the external world, and which imprison us' (O'Hara 2012, p. 118) and recognizes Ballard's identification of 'a designed psychosphere in which the 'death of affect' is one of the consequences of the psychological impasse in which our technological environments place us ( $\mathrm{O}^{\prime} \mathrm{Hara} 2012$, p. 118).' Yet as well as aiming to establish the complex and fluid intersections at play between psychopathology, affect theory, and social and online media, claims by the likes of Sampson, Ellis and Maddison demonstrate an intriguing theoretical counterpoint to Ballard's belief that a 'death of affect' was brought about by the same mediated world of rapid technological development, mass media and celebrity culture that led to him so astutely 'predicting' social media over forty years ago. For Ballard, such an affectless realm offered fertile breeding ground for thrilling, dangerous and necessary senses of deviance and perversity—something which represents another noteworthy comparison in light of Rottenberg and Johnson's affective approach to treating psychopathology. As such, this article will investigate how effectively Ballard's prescient thinking both reaffirms and challenges these discourses and theoretical approaches. To do this, it will draw upon some high-profile instances involving YouTubers and internet celebrities that not only demonstrate a marked, wilful deviance, but reveal much about the collapse of any pre-existing distinctions between private and public lives, and online and offline selves, in which a new psychopathology emerges. 


\subsection{Psychopathology and Affect}

The three objectives underpinning Rottenberg and Johnson's collection of studies on emotion and psychopathology are as follows: providing an overview of the most effective methodologies from clinical experts 'on major components of emotion, including self-report of experience, as well as behaviour, cognition, and physiological response' (Rottenberg and Johnson 2007, p. 7); identifying 'how emotion theory can inform treatment' (Rottenberg and Johnson 2007, p. 7) of psychopathology; lastly, illustrating 'how emotion research has the potential to provide understanding of key mechanisms in psychopathology (Rottenberg and Johnson 2007, p. 7)' The necessity of delving into emotion research is, they argue, due to a critical lacuna between the theoretical and the clinical when it comes to studying psychopathology. Introducing their collection of studies, they speak frankly about how 'scientific fragmentation has been acutely felt in the field of emotion, where there has been a wide gulf between "basic" research on normative emotion functioning and "applied" research on clinical disorders (Rottenberg and Johnson 2007, p. 4).' Responding to this gap in their field, their 'affective science' approach seeks to collapse this distinction with the hope that, in doing so, researchers and clinicians alike will begin to 'better understand both psychopathology and normal emotional variation (Rottenberg and Johnson 2007, p. 4)'.

The active and complex role that external environment plays in this interplay between emotion and psychopathology is crucial, to the point that it informs the definition of psychopathology upon which Rottenberg and Johnson foreground their work:

Although emotions can help us adapt successfully to the environment, psychopathology reveals the darker side of emotion ... On this darker side, emotional impulses are poorly tuned to the environment: They arise in the wrong contexts, build to the wrong intensity, and last for the wrong duration. When emotions go wrong, the consequences can be terrible ... emotional disturbance, in one form or another, is a central feature of psychopathology'. (Rottenberg and Johnson 2007, p. 3).

Additionally, the psychologists demonstrate an understanding of the fundamental complexities in attempting to bridge the gap between emotional, theoretical and clinical responses to psychopathology. The volatile, changeable nature of emotions and emotional disturbances are key here, as emotions 'can go wrong in so many ways, and these various forms of dysfunction are not easily described or arrayed into a meaningful scheme.' (Rottenberg and Johnson 2007, p. 4) This difficulty works right down to questions of definition around emotion, and how these questions in turn relate to an understanding of affect. Firstly, emotion:

is inherently a multifaceted construct. This is reflected both in definitions of emotion, which typically contain multiple elements such as subjective experience, cognition, behaviour, and physiology, as well as in wider discussions of emotion, which often draw on molar concepts such as culture, development, evolution, or socialization. (Rottenberg and Johnson 2007, p. 5).

Framing a study on bipolar disorder, Johnson, June Gruber, and Lori R. Eisner offer an interpretation of the differences between 'emotions' and 'moods', stating that while the latter tend 'to be long-lasting, diffuse, and not tied to a specific trigger, emotions are regarded as transient, acute responses to specific environmental stimuli that involve coordinated subjective, behavioural, and physiological components (Johnson et al. 2007, p. 124).' To add a further layer of complexity, affect is outlined by Humrichouse et al. as a state 'encompassing both emotions and moods. It is what one is experiencing or feeling, either pleasant or unpleasant, with varying levels of intensity, duration, and triggers or patterns of activation (Humrichouse et al. 2007, p. 14).' In addition to the aforementioned exploration into bipolar disorder, there are several other pertinent examples of specific psychopathological disorders that can be investigated using affective processes. One such example is Christopher J. Patrick's study into personality and neurobiology against the distinctive tropes of psychopathy, which is defined as: 
a severe personality syndrome that entails abnormalities in affective and interpersonal functioning accompanied by marked behavioural deviance. Psychopathic individuals appear charming and insouciant and exhibit a striking absence of guilt, remorse, or empathetic concern for others. These characteristics have led theorists to postulate a core underlying affective deficit in psychopathy (Patrick 2007, p. 125).

The idea of a 'core underlying affective deficit' existing in psychopathic individuals brings Ballard's connection between a collective 'death of affect' and psychopathology back into sharp focus. However, just as fundamental difficulties reveal themselves through the study of emotion, affect and psychopathology (working right down to a definitional level), Ballard's attitudes toward the causal relationship between a wholly mediated environment and psychopathology remain deliberately complex and changeable. Particularly intriguing are the ideas around individuals responding poorly to such environments and the subsequent differentiating from 'normal' emotional responses; the following sections of this article will explore how, in his fiction, Ballard playfully pushes the subjectivity of these terms.

\subsection{Affect and Online Participatory Media}

Reading an affective turn in studies of online participatory media as a clear-cut distinction from Ballard's ideas on the death of affect is not the aim here. The question of whether causality between psychopathology on one hand, and online and social media on the other, arises from a death of affect or an overabundance of negative affect is a hugely complex one. This is not least because online media does not exist in isolation as a technological development. It is not even merely because online media is actively and perniciously connected to celebrity culture and consumption, but rather because it is fundamentally redefining these aspects of the contemporary mediated landscape as well as also redefining traditional notions of family, and boundaries between public and private spaces.

The central role online media plays in the construction and very basis of the contemporary mediated world is paralleled by the inseparability of affect and social and online media. In charting this parallel, Ian Tucker hints at the psychological ramifications of such developments:

Digital media play an increasingly active role in conditioning the environmental contexts of psychological life. Media act as the environmental side of the "more than one" reality of subjective life. Therefore, emotions are becoming with digital media rather than being controlled and dominated by them [original emphasis]. This is a useful conceptual development because it provides a new perspective to digital media analysis in relation to affect and emotion. We see that living with digital media is by definition affective [original emphasis] (Tucker 2018, pp. 39-40).

If psychopathology is most likely to stem from a negative or poorly-tuned response to this digitally-mediated, affective environment, it does not take too much of an imaginative leap to consider how affect is directly linked to the social and cultural expectations of participation in this environment. This could be through Tweeting, Instagramming, or even heeding YouTube's encouragement to 'Broadcast Yourself'; however, the power of the dopamine hit of online validation can, almost imperceptibly, transform into distinctly negative affective responses. Ellis and Maddison put it bluntly: due to 'the overwhelming amount of participation on social media, it is not surprising at all that it often invokes insecurity and anxiety (Ellis and Maddison 2018, p. 99).' Rebecca Coleman riffs on the pressures associated with this level of participation by unpacking the self-descriptions of Facebook, Twitter and Instagram as a method of framing these platforms as 'hyper-connected, always on, affective and non-representational, and involved in re-working boundaries between production and consumption, and between temporalities and spatialities (Coleman 2018, p. 67)'.

These pressures to interact and participate- to throw oneself into this 'hyper-connected, always on' environment-are heightened in light of online celebrity culture. Greg Singh has undertaken excellent work exploring the intersection between 'celebrity, technology and selfhood in popular 
culture' (Singh 2017, p. 169), and the following discussion will follow his lead by taking YouTube as the platform from which to most effectively discuss online celebrity and its associated psychopathologies. In Singh's view, the video-sharing website functions 'as the popular media platform for online celebrity existing today' [original emphasis] (Singh 2017, p. 169), and as such forms the basis for his 'post-Jungian' approach to analysing the behaviour of online celebrities in addition to that of the fans and detractors of those celebrities. Tellingly, the most striking way in which Singh posits celebrity culture and celebrity interactions have been redefined by platforms like YouTube has a distinctly psychopathological edge to it:

Perhaps the most productive tension in the context of YouTube personalities and celebrity cultures is the blurred distinction between public and private in the identification, construction and mobilisation of the self. The immediacy and sheer speed of exchange, amplified through emotionally-charged celebrity culture, and engaged with by consumers of popular culture who are not only fans (and anti-fans, haters) of the celebrity figures themselves, means that consumer-users tend to be adept with the discourses featured in the communicative practices of platforms. Things tend to escalate very quickly under such intense circumstances (Singh 2017, p. 175).

Singh's ideas on the 'productive tension' inherent in the construction of the self are evocative of Gasiorek's reading of Ballard's late texts, which themselves 'describe a subjectivity so thoroughly imbricated in technological networks of power that no meaning exists in the conception of an authentic self (Gasiorek 2005, p. 208).' This 'productive tension', and its emergence in the collapsing of distinctions between public and private spaces, has also long been a consideration of theoretical work on YouTube and video-sharing platforms. Investigating the still-formative platform back in 2009, Michael Strangelove was quick to realise the new duality that could and would come to define the formerly private space of the family home, as well as the profound psychological repercussions of this. Indeed, Strangelove's opening gambit in his chapter on 'The Home and Family on YouTube' - the proclamation that the 'first studio of this mass age of amateur video is the home' (Strangelove 2010, p. 41), and that 'the domestic geography of the home forms part of the unconscious of this new mode of mass moviemaking' (Strangelove 2010, p. 41) -reads as positively Ballardian. As a prolific YouTuber himself, Strangelove demonstrates a clear recognition of the 'highly seductive' (Strangelove 2010, p. 47) allure of YouTube. Again, it is the participatory aspect of this new media that Strangelove realises is so powerful; he outlines how the validating prospect of having videos re-blogged or commented on contributes to an overwhelming 'aesthetic of attraction' (Strangelove 2010, p. 47). Yet despite-or perhaps because of-his early engagement with and immersion into the world of YouTube, Strangelove's work ultimately strikes a distinct note of caution, particularly with regard to this sudden blurring between private and public selves, asserting that all too often the practice of uploading home movies descends into a 'disturbing and harmful form of media practice' (Strangelove 2010, p. 52). Like Ballard, he cites the deviant possibilities of the technology as central to the formation of his views:

YouTube is a new form of looking glass; it enables others to apply deviant labels to anyone, which may lead individuals to internalize those labels. Such attitudinal changes in a person's self-concept may be temporary or permanent. It is not yet certain if the looking-glass effect occurs through the Internet's mediated social environments (Strangelove 2010, p. 58).

Strangelove's 2009 assessment is necessarily speculative, as well as remaining free from any Ballardian thrill of being through this particular looking glass. However, the 2017 controversy surrounding the DaddyOFive YouTube channel illuminates the theories of Strangelove and Singh. DaddyOFive centred on Maryland parents Michael and Heather Martin, and the 'pranks' that they and their four oldest children would periodically play on their unsuspecting youngest child, Cody. On one level, the DaddyOFive videos are clearly representative of Strangelove's early concern around how collapsing public and private spaces and selves were already constituting a 'dangerous and harmful 
media form of media practice', as in reality the 'pranks' played on Cody amount to little more than emotional and physical abuse.

Moreover, the nature of the discourse that played out in the wake of the DaddyOFive 'pranking' videos represents a typical example of the 'positive tension' that Singh originally formulated as a theoretical response to the similarly fractious \#GamerGate scandal. Rapid, emotionally-charged responses between fans of the Martins' channel (of which there were many), the conflation of those who showed concern for Cody Martin's wellbeing with so-called 'haters', as well as video responses from other prominent YouTubers like Philip DeFranco (2017) and the Martins themselves ratcheted up the intense emotional responses and brought into focus the ways in which the Martins' family unit was constructed and put on display for millions of viewers. Indeed, the power dynamics on display in the DaddyOFive videos-the parents and older siblings as perpetrators of the 'pranks'; the youngest child as perpetual victim of them-offer ample reason for Strangelove's cautious uncertainty of the looking glass effect of this mediated environment. Yet if 'it's just a prank, bro' (DeFranco 2017), as Michael Martin claims, then any long-term, real-world emotional consequences should not be an issue. Again, Singh tackles this question of the social and contextual murkiness of this section of online media culture by focusing on the:

treatment of performativity offered in any analysis of online social interactions where real-world consequences pertain. Through what might be described as a proxemics of YouTube and other platforms where social interaction of this kind proliferates, social psychology approaches serve to illustrate the complexities of social context cues, level of communication fidelity, non-verbal communications clarity, absence of eye-contact in non-visual communications forms, and the exaggerated effect of responsibility deferral through deindividuation (Singh 2018, p. 107).

The significance and complexity of these various and often incredibly subtle forms of technological communication can be traced right back to Gregory Bateson's concept of the double bind. Dan O'Hara describes the 'mutually contradictory messages' that are fundamental to any power dynamic in which a subject finds themselves psychologically trapped, by something such as familial ties: 'one of these messages is verbal, usually an injunction not [original emphasis] to do something, with an implied or explicit threat. The other message is more abstract, contextual, perhaps an intimation of love, and is non-verbal (O'Hara 2012, p. 108)'. Consequently, the Martins' video response to their 'haters' (clips from which are included in DeFranco's subsequent response to that video; the DaddyOFive channel is defunct as of July 2018) provides a further rich example of the way in which a typical, supposedly loving family unit is constructed for the camera as a means of attempting to negate any real-world psychological consequences, and defer any responsibility. Curiously, the evidence the Martins provide as a means of this deferral of responsibility and reinforcing of the family unit is the number of gifts and merchandise they are inundated with from fans; the belief in the power of these gifts hints at the curious role that a conspicuous consumption plays in these online social interactions and controversies.

For all that online media are redefining notions of celebrity, psychopathologies can also emerge as a consequence of hegemonic ideas around celebrity continuing to exist on these newer media platforms. Sophie Bishop takes the UK beauty vlogging industry as an archetype for this continuation:

The "A List" vloggers are overwhelmingly white, young, conventionally attractive and middle class. They have successfully negotiated the YouTube algorithm and gained millions of loyal subscribers on the video sharing platform. They are the visible girls: luminescent on the YouTube platform, on billboards paid for by YouTube, in magazines and L'Oreal commercials (Bishop 2018, p. 122).

Bishop convincingly argues how the algorithmic upholding of conventional ideals of fame and celebrity directly juxtaposes the suggestion of YouTube as a truly participatory online platform, as 'by definition, participatory media allows any user to design, create and publish their diverse content 
(Bishop 2018, p. 124).' Despite that principle, in the case of monetised YouTube channels, 'a small number of A List participants are promoted by a platform's algorithms, with the majority of users relegated to the long tail of backwater search results' (Bishop 2018, p. 124). Inevitably there are negative consequences for those users who do not conform to such ideals, as 'YouTube's algorithmic signals punish deviant content, straying from their commercial genre, and this too is a form of symbolic violence (Bishop 2018, p. 125)'.

The question that Bishop's assessment raises centres upon what happens when a user whose channel and content do not conform to these algorithmic and hegemonic requirements opts to respond in a way that shatters distinctions between the online and external worlds. In April 2018, YouTuber Nasim Najafi Aghdam opened fire at YouTube's headquarters in San Bruno, California, injuring three employees before turning her gun on herself. Aghdam's actions were motivated by the demonetisation and apparent censorship of her channel; as well as the racial undertones in YouTube's algorithm, recurring themes in her videos, such as images of extreme animal cruelty as a means of advocating animal rights and veganism, corroborate Bishop's point on the symbolic violence perpetrated by YouTube against seemingly 'deviant', uncommercial content.

\subsection{J. G. Ballard: Predicting the Psychopathology of Social Media?}

The fear and total unwillingness to tolerate anything that has even a hint of deviance to it that Sophie Bishop articulates so well within the framework of YouTube's insidious algorithms is, for Ballard, characteristic of post-war Western society. If one were to read Nasim Aghdam as a Ballardian figure based on this, then Ballard's assessment of the Baader-Meinhof's motives becomes highly revealing:

If you're brought up in one of these suburbs around a German city, where nothing is ever allowed out of place, where because they were so terrified by the experiences of World War II and the Nazi epoch, they'd gone to any length to make certain everyone is happy, everyone in school or kindergarten is dutifully equipped so there would be no deviance and no problems later. If you have a world like that, without any real kind of freedom of the spirit, the only freedom to be found is in madness ... in a completely sane world, madness is the only freedom! (Ballard 2012e, p. 154).

For Ballard, the embracing of madness reveals a profound sense of paranoia and desperation, but for all that, the wilful deviance that comes with embracing madness is hugely liberating and vitally necessary. In an affective, psychopathological sense, this kind of deviant, extremely violent response might well represent what Rottenberg and Johnson define as a poor emotional response to one's external environment. Ballard, however, is decidedly more ambiguous in how he views such psychopathological tendencies. Even though Ballard believes 'all we've got left is our own psychopathology. It's the only freedom we have-that's a dangerous state of affairs' (Ballard 2012a, p. 400), psychopathology is yet becoming 'the last repository of the human imagination ... [it] should be kept alive' (Ballard 2012d, p. 229). The attendant senses of very real danger and necessary imaginative capabilities are inseparable.

The technological, media and communications landscape is, of course, crucial to the development and exploration of Ballard's ideas and the complexities inherent within them. Speaking in 1979, Ballard's views on the possibilities that could be realised through technological and media innovations reveal similarities to Rottenberg and Johnson's concerns around poor individual responses to external environments:

with the new multimedia potential of your own computerised TV studio, where limitless simulations can be played out in totally convincing style, one will be able to explore, in a wholly benign and harmless way, every type of impulse-impulses so deviant that they might have seemed, say to our parents, to be completely corrupt and degenerate ... we'll shortly be moving into a realm where we will be able to take for granted the existence of 
these seemingly deviant interests and through the limitless powers of our home computers and TV we will be granted universes of experience which today seem to belong to the dark side of so-called civilised behaviour (Ballard 2012b, pp. 128-29).

The enticing possibility of moving to the dark side offers an inversion of Rottenberg and Johnson's insistence that the 'darker side of emotion' is symptomatic of psychopathology and would require treatment. This continued exploration of the compelling, deviant potential in embracing 'the dark side of so-called civilised behaviour' defines Ballard's later novels and their psychopathic anti-heroes. In Cocaine Nights (1996), Bobby Crawford continues the blueprint set out by Vaughan; Ballard (1997b) describes the two as 'deviant messiahs. They're sort of well-intentioned psychopaths. They're public-spirited psychopaths, a very curious blend. They genuinely want to do good and show people the truth (Ballard 2012c, p. 326)'.

Ballard states that one of the central aims of his later fiction was 'exploring the psychopathology of everyday life and trying to uncover those secret engines which keep us moving (Ballard 2012a, p. 397).' Published a decade prior to the inception of YouTube, the huge significance placed upon participating in amateur and home filmmaking in Cocaine Nights is particularly forceful. Moreover, the similarities between these yet-to-come online media platforms and the Laingian everyday psychopathology that plays out in Ballard's novel is illuminated by Singh's theories around the ways 'social media platforms, although pregnant with affirmative possibilities for political transgression, action and subversion, tend also to exacerbate the otherwise "normal" pathological split between an "inner self" and a generalized deadness of the embodied "false-self"' (Singh 2018, p. 108).

The sweltering, stifling backdrop of Estrella de Mar and the expats languishing within it provide the perfect testing ground for an experiment on how active participation in home moviemaking and this everyday psychopathology become thrillingly yet dangerously intertwined. Charles Prentice's initial impressions of the holiday resort set up Estrella de Mar as a necessary foil, through 'the timelessness of a world beyond boredom, with no past, no future and a diminishing present. Perhaps this was what a leisure-dominated future would resemble? Nothing could ever happen in this affectless realm, where entropic drift calmed the surfaces of a thousand swimming pools (Ballard 1997b, p. 35).' Just as Rebecca Coleman notes how social networking sites redefine understood temporalities as a way of cranking up the pressure to remain 'hyper-connected, always on', so the initial timelessness of Estrella de Mar is directly linked to its affectlessness. With this in mind, Bobby Crawford enters the novel as an embodiment of the pressures associated with online participatory media and its attendant psychopathologies. If the latter can be read through his wonderfully mindless criminality, Paula Hamilton most effectively recognises the importance of active participation that he demands: 'You're used to being an observer, and Bobby Crawford likes everyone to take part (Ballard 1997b, p. 200).' As the plot develops, Prentice is confronted with what participation in the new life of Estrella de Mar entails, and learns the centrality of video to this:

Crawford closed his eyes to the thought. 'That's not the kind of club I have in mind. People have to learn to switch off their TV sets. I want a club where people make their own films, learn how to storyboard a narrative, how to handle close-ups, dollies, pans and tracking shots. Film is the way we see our world, Charles' (Ballard 1997b, p. 261).

In addition to recalling the definition of a psychopath outlined by Christopher J. Patrick, Crawford plays a crucial role when it comes to Ballard's reworking of the idea that embracing the darker side of one's emotions and urges represents a 'poor' or socially unacceptable response to a given external environment. Here, the darker side of emotion is what allows characters to transcend their timeless, affectless realms in ways that are equally exhilarating and deviant. Additionally, Crawford's convincingness in his role of the everyday, benevolent psychopath marks him out as a precursor to the adeptness with which-as Singh argues-online celebrities and other users participate in and manipulate discourses and situations on online media platforms. 


\subsection{The Intensive Care Unit}

Ballard's prediction regarding the intrinsically 'benign and harmless' nature of online interactions can, with the benefit of a 2019 perspective, be undermined with relative ease (and all too many examples); indeed, Greg Singh's nuanced point on the dangerous and damaging murkiness between online and external social conventions works well to this effect. Nevertheless, the complexities and changeability of Ballard's thinking means any potential prescience should not be dismissed so quickly. 'The Intensive Care Unit' not only offers a playful engagement with the idea of 'benign and harmless' online relationships, but also more than an insinuation that Ballard maintains a full awareness of the dangers, as well as the possibilities, that arise when all sense of the affective distinctions between screen-based and sensuous worlds come crashing down.

An overwhelming sense of pride and hope emanates from the narrator and patriarch of 'The Intensive Care Unit' when he muses over the impending final, fatal conflict between himself, his wife and children. He shows an immediate awareness that 'this film will be the ultimate home movie, and I only hope that whoever watches it again will gain some idea of the immense affection I feel for my wife, and for my son and daughter, and of the unique affection that they, in their unique way, feel for me (Ballard 1994, p. 195).' The sentiment feels disconcertingly close to that expressed by Michael Martin, who utilised a crucial part of the discourse of an online scandal - the video response to those he deemed his 'haters' - to reaffirm his love for his family (DeFranco 2017). Not only this, the narrator broaches the central, unanswered, question that dominates Ballard's short story: just who is watching this battle play out? It hints at the existence of a potential audience of millions-as the DaddyOFive YouTube channel had-exploring their own psychopathological urges through the 'intensive care unit' of this one family. This uneasy feeling is compounded by the narrator's acute awareness of the necessary performativity and stylization of this final scene, and the role that the video camera plays within this.

The immersion of himself and his family into this immediate, violent and overtly sensory and emotional world ostensibly represents a complete departure from the narrator's previous life. Even if it is at an unconscious level, however, it also demonstrates the desperate necessity of getting beyond an entirely mediated, affectless existence. The narrator's first, abortive meeting with his wife prompts the-too late-realisation of the distinctions that had existed between screen-based and external, sensory worlds. More than that, though, the narrator's previous existence in a wholly mediated, televisual realm can now only be defined in relation to the grotesque, perverse intricacies of human physicality and directly experienced emotion:

True closeness, I now knew, was television closeness-the intimacy of the zoom lens, the throat microphone, the close-up itself. On the television screen there were no body odours or strained breathing, no pupil contractions and facial reflexes, no mutual sizing up of emotions and advantage, no distrust and insecurity (Ballard 1994, pp. 203-4).

The realisation undermines the narrator's renowned paediatric work up until that point, in which he has established the family unit as a 'fundamental unit ... of intensive care' (Ballard 1994, p. 201) through urging 'the installation of more cameras throughout the homes of family members' (Ballard 1994, p. 201). His thesis is that the ubiquitous presence of the camera will culminate in a 'liberating affectlessness [which] allowed those who so wished to explore the fullest range of sexual possibility and paved the way for the day when a truly guilt-free sexual perversity and, even, psychopathology might be enjoyed by all (Ballard 1994, p. 199).' This theoretical standpoint offers a notably similar-and as convincing-sentiment to the suggestion that the playing out of family 'pranks' and the online distribution of formerly private moments will have no bearing or consequences on the external world and its social contexts.

As the story closes, we find the narrator 'smiling at them affectionately, rage thickening the blood in my throat, I am only aware of my feelings of unbounded love (Ballard 1994, p. 205).' Ballard skilfully and playfully takes the narrator's previous thesis apart through the total, irreparable 
breakdown of the family's distinct screen-based relationships to one another. The intense, ambiguous emotional state of the narrator as the story ends paints an extremely vivid fictional picture of the emotional disturbances that underpin Rottenberg and Johnson's definition of psychopathology. It also foreshadows predictions from the likes of Strangelove by providing a prescient awareness of why the home movie would go on to become one of the most potentially damaging yet compelling - and crucially, extremely popular-genres of online video in the twenty-first century.

\section{Conclusions}

Everybody will be doing it, everybody will be living inside a TV studio. We're all going to be starring in our own sit-coms, and they'll be very strange sit-coms too, like the inside of our heads (Ballard 2012e, p. 155).

The starring roles that we all play in our own 'very strange sit-coms' are not limited to sharing videos of ourselves online, but rather they incorporate mass participation in online and social media discourses more broadly. Ballard goes further than offering a neat prediction of the front-of-house features of various social media sites; instead, he captures the curious dynamic between the collective fascination with online media and the intense social and cultural pressure to participate, as well as the psychopathological ramifications of this dynamic. Even with an understanding of the negative affect that constitutes a huge part of online media, Darren Ellis and Stephen Maddison acknowledge that:

Utility and enjoyment seem to outweigh the insecurity and anxiety that social media can provoke. The affective atmospheres of social media that contain insecurity and anxiety among many other affects do little to perturb participation. We love it! The trade-off seems worth it. Or perhaps there seems so little that can be done to stop it, we might as well join in, as avoidance may be even more difficult and dangerous (Ellis and Maddison 2018, p. 100).

The question of whether various forms of online media contribute to a contemporary death of affect, or whether they serve to drag us away from a mediated, affectless realm remains unsurprisingly unclear, given the complexity of Ballard's own views on the interplay between technology, affect and psychopathology. On one hand, the promises of harmlessly indulging in one's most deviant impulses without any external social or sensory consequences, or transcending hegemonic celebrity culture through these new platforms can fall apart with extreme results. The cases of DaddyOFive and Nasim Aghdam reveal the psychopathologies that emerge upon the complete blurring of online and external selves. Indeed, media coverage of Aghdam in the wake of the YouTube shooting painted a portrait of an eccentric, deranged individual with an intense hatred of YouTube; the similarities between Kingdom Come's (2006) Duncan Christie and his attitudes towards the MetroCentre felt chillingly apparent.

For all of these psychopathological pitfalls, however, Ballard fixates upon the thrilling, deviant potential of ubiquitous video sharing in Cocaine Nights and 'The Intensive Care Unit'. In these texts, participation in a hyper-connected form of moviemaking is put forward as the only chance to escape the eternal tedium of our contemporary mediated world. For all of the paranoid, desperate violence and sexual deviance that may arise, it is left to a note from Ballard's ultimate treatise on the media landscape of the twentieth-century—The Atrocity Exhibition (1970) — to have the final word: 'given the unlimited opportunities which the media landscape now offers to the wayward imagination, I feel we should immerse ourselves in the most destructive element, ourselves, and swim (Ballard 2014, p. 37)'.

Funding: This research received no external funding.

Conflicts of Interest: The author declares no conflict of interest. 


\section{References}

Ballard, James Graham. 1994. Myths of the Near Future. London: Vintage.

Ballard, James Graham. 1997a. A User's Guide to the Millennium. London: Flamingo.

Ballard, James Graham. 1997b. Cocaine Nights. London: Flamingo.

Ballard, James Graham. 2012a. Chris Hall: 'All we've got left is our own psychopathology': J. G. Ballard on Millennium People. In Extreme Metaphors: Interviews with J. G. Ballard 1967-2008. Edited by Simon Sellars and Dan O'Hara. London: Fourth Estate.

Ballard, James Graham. 2012b. Christopher Evans. The space age is over. In Extreme Metaphors: Interviews with J. G. Ballard 1967-2008. Edited by Simon Sellars and Dan O'Hara. London: Fourth Estate.

Ballard, James Graham. 2012c. Damien love. 'Kafka with unlimited Chicken Kiev': J. G. Ballard on Cocaine Nights. In Extreme Metaphors: Interviews with J. G. Ballard 1967-2008. Edited by Simon Sellars and Dan O'Hara. London: Fourth Estate.

Ballard, James Graham. 2012d. Solveig nordlund. Future now. In Extreme Metaphors: Interviews with J. G. Ballard 1967-2008. Edited by Simon Sellars and Dan O'Hara. London: Fourth Estate.

Ballard, James Graham. 2012e. V. Vale: Interview with JGB. In Extreme Metaphors: Interviews with J. G. Ballard 1967-2008. Edited by Simon Sellars and Dan O'Hara. London: Fourth Estate.

Ballard, James Graham. 2014. The Atrocity Exhibition. London: Fourth Estate.

Bishop, Sophie. 2018. \#YouTubeanxiety: Affect and anxiety performance in UK Beauty Vlogging. In Affect and Social Media. Edited by Tony D. Sampson, Stephen Maddison and Darren Ellis. London: Rowman and Littlefield.

Coleman, Rebecca. 2018. Social media and the materialisation of the affective present. In Affect and Social Media. Edited by Tony D. Sampson, Stephen Maddison and Darren Ellis. London: Rowman and Littlefield.

DeFranco, Pillip. 2017. WOW ... We Need to Talk About This ... 2017. Available online: https:/ / www.youtube. com/watch? $\mathrm{v}=$ fvoLmsXKkYM (accessed on 29 November 2018).

Ellis, Darren, and Stephen Maddison. 2018. Introduction to Part III. In Affect and Social Media. Edited by Tony D. Sampson, Stephen Maddison and Darren Ellis. London: Rowman and Littlefield.

Gasiorek, Andrzej. 2005. Contemporary British Novelists: J. G. Ballard. Manchester: Manchester University Press.

Humrichouse, John, Michael Chmielewski, Elizabeth A. McDade-Montez, and David Watson. 2007. Affect assessment through self-report methods. In Emotion and Psychopathology. Edited by Jonathan Rottenberg and Sheri L. Johnson. Washington: American Psychological Association.

Johnson, Sheri L., June Gruber, and Lori R. Eisner. 2007. Emotion and bipolar disorder. In Emotion and Psychopathology. Edited by Jonathan Rottenberg and Sheri L. Johnson. Washington: American Psychological Association.

Lewis, Luke. 2013. J. G. Ballard Predicted Social Media in 1977. Available online: https:/ /www.buzzfeed.com/ lukelewis/jg-ballard-predicted-social-media-in-1977 (accessed on 29 November 2018).

O'Hara, Dan. 2012. Reading posture and gesture in Ballard's novels. In J. G. Ballard: Visions and Revisions. Edited by Jeannette Baxter and Rowland Wymer. Basingstoke: Palgrave Macmillan.

Patrick, Christopher J. 2007. Affective processes in psychopathy. In Emotion and Psychopathology. Edited by Jonathan Rottenberg and Sheri L. Johnson. Washington: American Psychological Association.

Rottenberg, Jonathan, and Sheri L. Johnson. 2007. Introduction: bridging clinical and affective science. In Emotion and Psychopathology. Edited by Jonathan Rottenberg and Sheri L. Johnson. Washington: American Psychological Association.

Sampson, Tony D., Stephen Maddison, and Darren Ellis. 2018. Introduction: On affect, social media and criticality. In Affect and Social Media. Edited by Tony D. Sampson, Stephen Maddison and Darren Ellis. London: Rowman and Littlefield.

Sellars, Simon. 2013. No Original Response: J. G. Ballard predicts Social Media, CCTV, Reality TV. Available online: http:/ / www.ballardian.com/j-g-ballard-predicts-social-media-cctv-reality-tv (accessed on 29 November 2018).

Singh, Greg. 2017. YouTubers, online selves and the performance principle: Notes from a post-Jungian perspective. CM: Communication and Media 11: 167-94. [CrossRef]

Singh, Greg. 2018. Wupocalypse now: supertrolls and other risk anxieties in social media interactions. In Affect and Social Media. Edited by Tony D. Sampson, Stephen Maddison and Darren Ellis. London: Rowman and Littlefield. 
Strangelove, Michael. 2010. Watching YouTube: Extraordinary Videos by Ordinary People. Toronto: University of Toronto Press.

Tucker, Ian. 2018. Digitally mediated emotion: Simondon, affectivity and individuation. In Affect and Social Media. Edited by Tony D. Sampson, Stephen Maddison and Darren Ellis. London: Rowman and Littlefield. 